\title{
Linx
}

Revue des linguistes de l'université Paris X Nanterre

$8 \mid 1996$

Du dire et du discours

\section{Le mot au risque du dictionnaire. Sommes-nous tous des étrangers de la langue maternelle?}

Francine Mazière

\section{OpenEdition}

Journals

Édition électronique

URL : http://journals.openedition.org/linx/1105

DOI : 10.4000/linx.1105

ISSN : 2118-9692

Éditeur

Presses universitaires de Paris Nanterre

Édition imprimée

Date de publication : 1 septembre 1996

Pagination : 13-23

ISSN : 0246-8743

\section{Référence électronique}

Francine Mazière, « Le mot au risque du dictionnaire. Sommes-nous tous des étrangers de la langue maternelle? », Linx [En ligne], 8 | 1996, mis en ligne le 24 juillet 2012, consulté le 20 avril 2019. URL http://journals.openedition.org/linx/1105 ; DOI : 10.4000/linx.1105

Ce document a été généré automatiquement le 20 avril 2019

Département de Sciences du langage, Université Paris Ouest 


\title{
Le mot au risque du dictionnaire. Sommes-nous tous des étrangers de la langue maternelle?
}

\author{
Francine Mazière
}

"Je fis remarquer à Gertrude les sonorités différentes des cuivres, des instruments à cordes et des bois. » Gide

Petit Robert 1990, article instrument, I,2

1 Pendant longtemps j'ai regardé le dictionnaire monolingue comme une entreprise un peu insensée et cependant indispensable à une communauté, dès qu'elle a conscience d'user d'une langue dite internationale.

2 Sur le plan pratique, dans leur grande majorité, les définitions ne seront jamais consultées (d'où les dictionnaires spécialisés, mieux ciblés et d'ailleurs plus anciens). Dans l'ordre du politique, on peut constater qu'il n'existe pas de dictionnaire monolingue du kaingáng, ou de quelqu'autre "petite langue", mais pas non plus de dictionnaire monolingue de l'espagnol ou du français avant les consistances étatiques du XviI ${ }^{\mathrm{e}}$ siècle.

Pourtant, dans leurs justifications des premiers monolingues, tous les auteurs avancent dans les préfaces une prétention pratique de service, d'utilité pour «les étrangers qui aiment notre langue 'et même' les Français qui doutent ", comme l'annonçait déjà le premier Dictionnaire de l'Académie, en 1694 (désormais DA). Et sans doute est-ce ainsi que l'entend le public, qui achète.

4 Je voudrais ici m'arrêter sur un aspect restreint de ce programme pragmatique d' apprentissage qu'annoncent les monolingues, celui qui touche à la reconnaissance du mot compris comme unité linguistique déterminée d'abord par la relation forme/sens. Ceci demande une analyse du dictionnaire comme discours historique et comme dispositif d'observatoire du sens.

Bien entendu, en termes quantitatifs, nous pouvons lire le dictionnaire de 7 à 77 ans et y apprendre tous les jours de nouveaux mots, d'autant plus que le TLF' ${ }^{1}$ élaboré à partir de 
90 millions d'exemples, et le GLLF avec ses 163270 entrées (le PR en a environ 60 000) avouent leur incomplétude. Mais le quantitatif n'est pas ce que visaient les programmes des premiers monolingues puisque le fameux Dictionnaire de l'Académie se construit explicitement sur l'exclusion: exclusion des termes techniques (à l'exception de ceux qui ont donné des métaphores), exclusion des termes bas (mais pas grossiers au sens actuel), exclusion des vieux mots et des néologismes. Cependant, dans la tradition française, il est celui qui insiste le plus sur sa valeur pragmatique d'apprentissage de «La Langue Française $»^{2}$.

6 Écartons encore une justification facile de ce programme : il s'agissait, au XVII ${ }^{\mathrm{e}}$ siècle, de fixer comme bon usage le français de la Ville, minoritaire, la langue qu'écrivaient les écrivains et que parlaient les orateurs politiques et les dames. Ceci est vrai mais tout à fait insuffisant.

7 Devoir inventer un discours définitoire sur les mots les plus simples d'une langue ${ }^{3}$, et dans cette langue, c'est devoir jouer en permanence sur un dedans-dehors de la langue assez hasardeux mais contraint, et donc participer à l'invention de cette langue, en la triant, choisissant, arrêtant, jugeant, même sans jugement exprimé. En effet, si l'entrée se fait «en langue », par l'image graphique du signifiant (fonction autonymique), l'article s'organise «en discours", rédigé en langage ordinaire, soumis aux genres établis, aux habitudes de dire, parfois créateur de dires exorbitants, et cependant constitués par et constitutifs de la notion même de langue.

Ceci incite à user du dictionnaire comme d'un dispositif opaque certes, mais permettant néanmoins d'observer le statut instable des entités lexicales, entre langue, norme et discours. Pour cela, je développe trois petites études inspirées de travaux récents, et concernant ${ }^{4}$ :

- les bergers des bords de Seine,

- les cors de chasse du poète,

- les métallophones des grands magasins.

\section{1.}

9 Le xylophone, comme son nom l'indique aux hellénistes, est un instrument sonore en bois. Le dictionnaire peut en donner une définition décomposante: xylo + phone, traditionnellement appelée étymologique. Sans parler des problèmes que poserait la systématisation de lecture du procédé (le saxophone n'est pas un instrument sonore de Saxe mais fut inventé par monsieur Sachs), toute approche quelque peu historiciste du mot (c'est-à-dire qui descend le temps au lieu de le remonter comme le fait l'étymologie) pourra substituer une définition fonctionnelle tout aussi traditionnelle quand il s'agit d'outils. Par exemple : instrument de musique fait de lames de bois que l'on frappe avec deux petits marteaux.

10 Le xylophone est un instrument assez ancien et simple, devenu presque un jouet pour les enfants. Du temps où il était vraiment en bois, les lames étaient faites de bois précieux : bois de rose, palissandre. Mais les lois de l'évolution font qu'il y a aujourd'hui des xylophones dont les lames sont en métal. Le PR comme le DFC signalent les deux matériaux : bois ou métal.

Le nom est pourtant resté et personne ne pense plus au bois, sans particulière culpabilité. 
12 Voire. Les marchands (fabricants) de jouets ont de ces remords de scientificité remotivante qui peuvent faire rêver les parleurs puristes, pris entre l'usage et le «sens propre » mais submergés par le succès général des remotivations. A Noël, vous trouverez des métallophones dans les grands magasins. Complexe de modernisme? ou complexe de légitimité linguistique que n'éprouvent pas les lexicographes?

\section{2.}

Qui parle de cors de chasse ? Voici un « mot de dictionnaire » dont nous pouvons suivre la constitution dans un article du Petit Robert.

La tradition lexicographique est longue. Elle a peu à peu séparé des secteurs dans l'article: marques (de style ou de domaine), définitions, exemples (cités ou forgés), et locutions. Elle a aussi dû se dégager de la double tradition des bilingues et des dictionnaires de "façons de parler", qui ne posaient pas l'autonomie du mot mais privilégiaient les collocations, "mots qui vont ensemble», comme les définit le DA, dictionnaire qui les répertorie comme la norme du bien parler et les liste, en fin d'article ${ }^{5}$. Peu à peu, ces appariements quittent la fin d'article, où ne restent que quelques locutions figées. Les collocations sont alors employées, sous couvert de langue ordinaire, à des tâches techniques diverses. Elles peuvent apparaître comme façon de dire un domaine ( fabrique, en terme d'église/fabrique d'église, biniou, (rég): cornemuse bretonne) ou comme équivalent synonymique définitionnel (hautbois : cor anglais) ou comme usage discursif artificiel (trompe de chasse, les chasseurs disent trompe) ou comme liées à une tradition historique (trompettes turques et effets turcs dans l'orchestration). Ces exemples ont été explicités (Léon / Mazière 1996) et ne sont rappelés ici que pour situer notre cor de chasse.

L'article cor du DFC emploie cor de chasse en usage, en opposition, classiquement, à l'instrument d'orchestre ${ }^{6}$.

COR : Instrument de musique à vent, en cuivre, fait principalement d'un tube enroulé sur lui-même : Les cors d'un orchestre symphonique. Le cor de chasse ne comporte pas de clefs.

Soit maintenant les articles trompe et cor dans le PR, dont on extrait les collocations cor simple, cor de chasse, comme équivalent d'une définition (générique : cor + détermination : simple/de chasse) et / ou comme mot composé :

TROMPE... $\left(\mathrm{XVI}^{\mathrm{e}}\right)$ trompe de chasse, ou trompe, cor simple. - Petit instrument à vent très sommaire, servant à appeler. $\mathrm{V}$. Corne, cornet. Berger qui sonne de la trompe.

COR... Cor de chasse (lang. cour.) Les chasseurs disent Trompe. "J'aime le son du cor, le soir ou fond des bois. " (Vigny)

$\mathrm{Si}$ « cor simple » semble bien une définition dans TROMPE, Cor de chasse, comme trompe de chasse, trompe figurent en italique dans les articles. Cor de chassefonctionne-t-il comme lexème composé ou comme définition donnant un domaine d'usage ? Le choix de lecture est laissé au lecteur, semble-t-il. En effet, alors que trompe est donné comme équivalent synonymique de trompe de chasse, sans précision d'usage, cor de chasse est suivi d'une marque (lang. cour.) et d'un " commentaire » : les chasseurs disent trompe. Alors, qui dirait cor de chasse ? Le co-terme chasse est-il sous-entendu dans certains discours spécialisés, en particulier celui du domaine reconnu (on voit (entend) mal un chasseur demander son cor de chasse) ? Cor de chasse, trompe de chasse fonctionnent-ils comme collocations donnant le domaine sans être «naturellement » en usage ? Dans une énumération de catalogue, par exemple ${ }^{7}$. Autrement dit, le co-terme n'est-il que lexicographique, marqueur de domaine 
et parasite en discours ordinaire ? Un " mot de dictionnaire » en somme, de lexicographe et non de locuteur? La citation le laisserait penser, qui ne reprend pas la collocationlexème complète cor de chasse mais seulement cor: «J'aime le son du cor le soir au fond des bois. " (Vigny) ${ }^{8}$.

Mais reste l'autre poète, au cœur de la langue (ou dans un autre discours ?) :

Les souvenirs sont cors de chasse

Dont meurt le bruit parmi le vent

Apollinaire, Alcools, Cors de chasse

\section{3.}

Les bergers préfèrent le chalumeau. Et il n'est pas très facile de savoir ce qu'il faut entendre par là.

Lors du travail déjà cité sur les instruments de musique, nous n'avions pas relevé chalumeau, qui ne figure pas dans la liste des instruments à vent du Grand Robert. Cependant, les premiers monolingues comme les plus récents l'incluent dans leur nomenclature. Ainsi le PR90 :

CHALUMEAU n. m. (1464; chalumel, Xlle; bas lat. calamellus de calamus « roseau »). $\cdot 1^{\circ}$ Tuyau de roseau, de paille. V. Paille. $\cdot 2^{\circ}$ Mus. Flûte champêtre, simple tige percée de trous. V. Flûtiau, pipeau. $\diamond$ Registre grave de la clarinette. $\bullet$ $3^{\circ}$ Appareil qui $[. .$.

Le générique Flûte et le déterminatif champêtre posent problème. Cette définition était déjà celle du DA en 1694, mais, malgré l'ancienneté de l'objet, qui a peu évolué, sans doute n'avait-elle pas le même sens. Nous allons voir pourquoi.

Flûte est un bon candidat à devenir hyperonyme, dans l'exacte mesure où il entre en collocation de façon récurrente et diversifiée [PR90]:

FLUTE [flyt]. n. f. (Flehute, XIIe. Cf. prov. Flauto d'o. i.). • $1^{\circ}$ Instrument à vent formé d'un tube creux percé de plusieurs trous, ou de tubes d'inégales longueurs. Flûte de Pan, faite de roseaux d'inégale longueur. V. Syrinx. La flûte, instrument de musique traditionnel des bergers. Petite flûte en bois encore utilisée dans la musique militaire. V. Fifre. Flûte à l'oignon. V. Mirliton. La flûte enchantée, opéra de Mozart.Mus. Flûte traversière. Grande flûte. Petite flûte. V. Piccolo. Flûte à bec : ancien instrument. Spécialt. La grande flûte. - Loc. Être du bois* dont on fait les flûtes [...].

La similitude de l'énoncé descriptif pour flûte et chalumeau, à savoir, tube/tige percé(e) de trous n'implique cependant pas de renvoi à chalumeau dans l'article FLÛTE. Mais un curieux énoncé, au statut incertain (la présence du déterminant empêche d'en faire une sousentrée et il ne peut s'agir d'un exemple) signale La flutte, instrument de musique traditionnel des bergers.

Et si l'on revient à chalumeau les renvois à flutiau et pipeau développent la détermination « champêtre »:

FLUTEAU [flyto] ou FLUTIAU [flytjo]. n m (Flusteau XVI ${ }^{e}$ flaihutel, XIII ; de flehute « flûte »). $\cdot 1^{\circ}$ Petite flûte sommaire (chalumeau ${ }_{2}$ flageolet).

PIPEAU [pipo]. n nz. (1563, de pipe) $1^{\circ}$ Flûte champêtre (symbole de la poésie pastorale). V. Chalumeau. $\cdot 2^{\circ}$ Appeau $\left(1^{\circ}\right)$. [...]

Il semble donc que la détermination champêtre commence à prendre sens dans une mise en réseau avec sommaire, pastoral et peut-être de berger. 
21 aut, pour comprendre la confusion, se souvenir du traditionnel recopiage des dictionnaires durant trois siècles. Furetière, dont les définitions sont partout louées, définissait ainsi le chalumeau, dans son Dictionnaire Universel de 1690 :

Chalumeau: tuyau de blé.chalumeau se dit aussi d'un instrument de musique, champêtre, composé soit d'un, soit de plusieurs tuyaux de bled, soit de quelque autre matière déliée. Les chalumeaux ont servi de flûte à nos anciens et on en a fait de l'écorse d'un saule levée [...].

Le tuyau, caractéristique de la flûte, et surtout la dénomination champêtre sont donc dans le Furetière, comme dans le DA. Le plus intéressant n'est pas là mais dans le fabuleux exemple du même lexicographe :

Les bergers dans leurs églogues disent qu'ils chanteront les louanges de leurs bergères sur leurs chalumeaux.

Tout serait à commenter : le sujet au pluriel et le possessif de relation leurs bergères, le verbe déclaratif dire, le futur et surtout dans leurs églogues, imparable positionnement littéraire, qui n'a rien de rustique.

S'éclairent du même coup les réemplois de l'hyperonyme, flûte, pris dans une inversion par l'usage pragmatique («Les chalumeaux ont servi de flûte à nos anciens. ») et de la détermination, champêtre, sur laquelle porte la migration du sens.

Parce que le dictionnaire, sous ses allures benoîtement unifiantes, est un lieu de mise en contact d'univers discursifs disjoints, la polysémie s'y rencontre comme fonction de l'histoire des pratiques discursives.

Pour CHALUMEAU, les rédateurs ont conjoint le traitement analogique du générique (tuyau de paille), recopié du Furetière, et qui pourra s'inscrire dans le technique (tube de la musette, du biniou, tube de verre, appareil à souder...) et la dérive synonymique, par désuétude des pratiques: la pastorale, aristocratique au XVII ${ }^{\mathrm{e}}$ siècle, genre littéraire ${ }^{9}$, se rabat sur champêtre, qui induit du discriminant social, ou sommaire, qui deviendra même rustique (à la fois paysan et sommaire) dans le TLF. Un référent nouveau, suggérant du « réel » derrière le berger d'églogue, et partant, un autre référent, s'est glissé dans notre nouvelle lecture, inscrite dans notre nouvel espace-temps.

Et voilà pourquoi on trouve tant de pastorales et de bergers curieusement occupés dans nos dictionnaires modernes ${ }^{10}$.

Pour nous résumer, métallophone dit simplement le marquage du phénomène d'hypercorrection au niveau du mot, c'est-à-dire comment la légitimité du locuteur limite celle de la remotivation. Le dictionnaire résiste au néologisme en fonction de critères certes linguistiques (rejet assez général de la remotivation qu'on pourrait dire populaire), mais inscrits dans un groupe (ou des groupes) de locuteurs exclusifs.

Cor de chasse trace des limites dans la langue (et/ou dans le discours) entre mot et non mot. Le dictionnaire a le mérite d'exhiber le problème et cette entrée montre la confusion programmée, sans doute théorisable, entre définition et usage des locuteurs. En particulier, le rapport entre définition et domaine d'emploi autorise des taxinomies floues, voire des inventions discursives, non attestées (cf note 8) et, au plan linguistique, ne permet pas la caractérisation du mot composé par rapport au syntagme libre. Ainsi le dictionnaire est pris dans l'illusion de clarté de sa métalangue propre, ici, la marque de domaine, et des propriétés de la structure du GN français (en particulier la détermination) posé comme texte de définition ${ }^{11}$. 
Cameau est tout discours : le dictionnaire, en posant les attributs de la typicalité dans la définition et non dans la seule collocation, ou dans l'exemple, aplatit le programme narratif et historique promené par le mot, au point que le phénomène de condensation, propre à l'effet du glissement métaphorique, installe le contresens dans les co-termes (ici, berger et pastoral). Le dictionnaire, à l'intérieur de l'énoncé définitoire, gère mal l'historicité que lui transmet ce que j'appellerai les « liaisons » de mots, éléments mis en place discursivement, donc dans des espace-temps caractérisables, et devenus purement linguistiques en synchronie. Autrement dit, l'effet de déplacement sémantique, dans les espace-temps, des prototypies discursives.

Le mot, définitivement, n'est un objet de la langue que posé dans l'abstraction de sa signification et la matérialité morpho-phonologique de son signifiant ${ }^{12}$. Mais, en dictionnaire, il est d'abord de l'ordre du discours et relève de son analyse ${ }^{13}$.

Regardé de ce point de vue, il est même une vraie serpillière qui ramasse, garde et transporte ce qu'elle récolte dans les recoins de ses usages, qui ne perd pas ou peu mais attrape, par les structures langagières de l'association et de la constellation, de la détermination, de la classification et de la synonymie, sa face "trésor partagé ». A cause de cela même, le mot est toujours étranger au locuteur partiel que nous sommes. Le sens excède toujours parce que les mots ont entre eux des liaisons déterminées interdiscursivement, mais dont on oublie la détermination. Or, ce sont les liaisons des mots, manifestées dans l'énoncé phrastique (et non paraphrastique) de la définition, qui créent leurs référents langagiers. Avant même l'emploi en phrase, le mot est conditionné par des référents d'usage, ou des traces de référents constitués dans les pratiques discursives. Il est donc hétérogène et non pas seulement polysémique.La valeur saussurienne n'est peut-être pas que le différentiel entre mots mais aussi le différentiel à l'intérieur des mots, jamais fermés sur eux-mêmes, jamais pleins, jamais libres, pris dans leurs « liaisons » d'ordre langagier et historique.

Le dictionnaire a un sens comme outil linguistique ${ }^{14}$ dans l'exacte mesure où il a inventé une construction syntaxique, la définition, qui donne l'apparence d'une structure de signification et autonomise le mot. Par là, il pose le mot comme définissable, c'est-à-dire assimilable à une phrase de substitution (équivalence possible par le verbe être), elle même faite de mots, eux-mêmes ayant des définitions, etc.; aucune circularité dans ce procédé, seulement une circulation des sens qui manifeste cette banalité que le signe ne « correspond » pas à un référent mais a cependant un sens irréductible, garant de l'usage discursif, jamais fini mais jamais sans possibilité définitoire. Le dictionnaire monolingue fixe la langue, dit-on. En fixant un "prêt à parler " ${ }^{15}$, il ne désigne pas un item lexical mais un « segment de discours » ${ }^{16}$. Il n'y a pas de dictionnaire « de langue ». En ce sens, le dictionnaire monolingue est bien d'abord un outil de politique linguistique au sens le plus large. S'il est aussi un outil métalinguistique, c'est par l'invention de la forme de l'article (définition/citation, morphologie, étymologie, histoire, traitement de la polysémie), c'està-dire dans la manifestation du rapport sens/forme à partir d'un graphisme, l'autonymie de l'entrée. En ce sens, il est un observatoire qui configure quelques informations sémantiques et de construction, de telle sorte que l'analyse peut en donner des interprétations.

31 En nouant une jolie faveur au cou de chaque mot, la définition fait sans doute le contraire de ce qui paraît. Le dictionnaire déloge en permanence ce qu'il énonce, à savoir que la définition pourrait être autre chose qu'un discours excluant - par définition même - 
d'autres discours sur le mot. Pluralité et hétérogénéité comme nécessité, non comme faiblesse. Sinon pas de jeu, partant, pas de discours, et pas de langue.

La vogue des dictionnaires monolingues ne se dément pas. Cela se comprend. On peut s'asseoir dessus, le copier, le voler, parfois le consulter, et, plus délicieux et plus pervers, peut-être plus scientifique, le lire et proposer une analyse de son discours. Preuve que ce qu'il dit nous est extérieur, qu'il nous excède, en même temps qu'il nous insupporte, comme étant toujours à côté et toujours en deçà de l'attente démesurée de complétude qui nous hante.

\section{BIBLIOGRAPHIE}

\section{Ouvrages de référence pour le corpus}

Petit Robert, (1977) et (1990) Paris, Ed Le Robert

Dictionnaire du Français Contemporain, (1966) Paris, Larousse

FURETIÈRE, A. (1690) Dictionnaire Universel contenant généralement tous les mots François tant vieux que modernes et les termes de toutes les sciences et art., Rotterdam, Arnout et Reinier

Dictionnaire de l'Académie (1694) 2 vol. , Paris

Trésor de la Langue Française, CNRS, Gallimard, Paris

\section{Bibliographie}

AUROUX, S. (1992) Histoire des idées linguistiques T2, Liège, Mardaga.

- (1994) La révolution technologique de la grammatisation Liège, Mardaga.

COLLINOT, A. et MAZIÈRE, F. (1987) Un prêt-à-parler : le Dictionnaire Universel d'Antoine Furetière et sa postérité immédiate, le Trévoux. Une lecture du culturel dans le discours lexicographique, Lexicographica 3, pp. 51-75, Tübingen.

- (1990) Les définitions finalisées dans le Furetière. Où il est montré que l'hétérogénéité syntaxique des définitions a du sens. Dans J. Chaurand, F. Mazière, La définition, Paris, Larousse.

- (1993) Une autre lecture du Dictionnaire de l'Académie (1694) : valeur instrumentale du préconstruit en analyse de discours. Actes du colloque Parcours linguistiques de discours spécialisés Paris./Genève, Université de Paris III/Peter lang.

GOURDET, G. (1967) Les instruments à vent, Paris Que-sais-je ?, PUF.

HONEGGER, M. (1977) Science de la Musique, Paris, Bordas.

LÉON, J. et MAZIÈRE, F. (1996) Collocations et dictionnaires dans La locution : entre lexique, syntaxe et pragmatique Collection Saint Cloud, Publications de l'INaLF, Klincksieck, Paris. 
MAZIÈRE, F. (1995) Le Dictionnaire de l'Académie : un pacte linguistique. Construction de l'article de dictionnaire de langue. Actes du colloque sur le deuxième centenaire de l'Académie Française.

\section{NOTES}

1. TLF : Trésor de la langue française; GLLF : Grand Larousse de la langue française ; PR : Petit Robert 1990 ; GR : Grand Robert ; DFC : Dictionnaire du français contemporain.

2. Collinot/Mazière 1993.

3. «Elle [l'Académie] a donné la Définition de tous les mots communs de la Langue dont les Idées sont fort simples; et cela est beaucoup plus malaisé que de définir les mots des Arts et des Sciences dont les Idées sont fort composées ; Car il est bien plus aisé, par exemple, de définir le mot de Télescope, qui est une Lunette à voir de loin, que de définir le mot de voir; Et l'on éprouve même en définissant ces termes des Arts et des Sciences, que la Définition est toujours plus claire que la chose définie ; au lieu qu'en définissant les termes communs, la chose définie est toujours plus claire que la Définition ». Préface.

4. Travail sur les instruments de musique avec J. Léon, en partie publié (1995). Xylophone a été abordé à propos d'un travail pour un Livre des mots avorté.

5. Mazière 1996.

6. L'orchestre est devenu la référence obligée pour tous les instruments après les innovations du $\mathrm{XIX}^{\mathrm{e}}$ siècle (lien entre l'invention de l'orchestre et l'invention des clefs, pour beaucoup d'instruments à vent traditionnels qui ne reparaissent en concert dans leur forme ancienne que dans la musique baroque, la musique pastorale ou le folklore). La musique est un des domaines où la persistance des appellations est en permanence contrariée par la pratique sociale (la trompette est encore un « instrument militaire » dans Furetière (1690)) et par l'ethnocentrisme.

7. « da caccia» est une détermination courante dans le vocabulaire des instruments de musique en italien. Le terme peut être traduit, et produire alors un " mot de traduction ».

8. Le TLF sépare deux domaines constitués, chasse et musique, et n'emploie cor de chasse que sous une rubrique ajoutée : spécialement, avec cor de mer et cor des Alpes. Effet positif d'un traitement statistique sur corpus, qui évite la confusion entre item et classification mondaine.

9. Pastorale : «Genre dramatique qui relate les exploits amoureux de bergers et de bergères, situés dans un cadre naturel, un havre idyllique de bonheur et de paix, comme celui de l'Arcadie, où habite Pan, le dieu des bergères, et Alphée, celui des fleuves. La poésie de la pastorale est la première à s'agrémenter entièrement de musique, pour être chantée, dansée et non pas parlée » Dictionnaire de la Musique, Larousse, 1990.

Les pastorales sont intégrées dans les premiers opéras en Italie (Monteverdi) et en France (Lully). Pendant deux siècles (jusqu'à la Révolution) elles règnent sur la musique vocale profane et marquent la musique religieuse et instrumentale. Au Moyen Age, c'est une chanson allégorique, aristocratique, courtoise. En France, la pastorale devient un genre littéraire sans musique au début du XVIIe: roman pastoral d'Urfé, L'Astrée, puis poésie précieuse. Par ailleurs, les représentations sacrées, les mystères, les intermèdes de cour, l'opéra naissant obéissaient à certaines conventions en ce qui concerne les instruments. Les scènes infernales sont rendues par les cuivres graves, les percussions ; la terre par les violes, cithares, flûtes et trompettes ; les bruits de guerre par les trompettes et tambours; les évocations pastorales par les bois; les élans d'amour par les cordes ; les tableaux célestes par les luths, harpes et orgues.

10. Mais les ouvrages spécialisés participent de la même équivoque. Je donne ici des extraits de sources explorées par J. Léon.

Denis Levaillant, Corps Ecrit, 35, 1990 «Ses lettres de noblesse, la clarinette semble les avoir acquises quand il était encore temps : tout le monde s'accorde en effet pour attribuer au facteur Denner, de Nuremberg, l'invention de l'instrument européen moderne, vers 1700 . En réalité, 
Denner a inventé le bec, et a rajouté deux clefs au vieux chalumeau roturier. » [c'est moi qui souligne]

Le terme roturier est une amplification du décrochage vers la rusticité par oubli de l'aristocratique pastorale, et dit plus sur la séparation/classification des instruments en simples et sophistiqués.

Mais d'autres traces, autour de l'ambigu " pastoral », sont repérables, en locutions convenues :

Dictionnaire de la Musique, Larousse, 1990 : « Le chalumeau peut être considéré comme l'ancêtre de la clarinette. [É] Dans le langage courant, on parle aussi de chalumeau pour désigner un pipeau pastoral fait d'un tuyau de paille ou de roseau. »

- Honegger : «ce nom a été appliqué à divers instruments à anche [...] de façon générale, il désigne un pipeau pastoral fait à partir d'un épi de maïs, ou [...]»

- Goudet : «L'ancêtre européen de la clarinette est le pastoral chalumeau français du moyen âge et de la renaissance [...].»

11. La définition de nom fait un usage textuel d'une forme syntaxique, la forme GN, et exploite toutes les possibilités stucturales d'un GN français. Pour une analyse, Collinot/Mazière.

12. Depuis Saussure au moins, la linguistique sait que l'entité linguistique est construite. Si la grammaire scolaire $\mathrm{du} \mathrm{XX}^{\mathrm{e}}$ siècle a pu être critiquée, c'est pour sa pratique mécaniste des catégories comme évidences, et le dictionnaire, en se posant comme "recueil» a entretenu l'illusion de la « donnée » concrète.

13. On comprend dès lors que la relation définition/citation dans les dictionnaires soit à la fois passionnante et très irritante.

14. Notion proposée et théorisée par Sylvain Auroux, développée au sein de l'URA 381.

15. Collinot/Mazière 1987.

16. L'expression est reprise d'André Collinot. Papier de travail, CELEX. Non publié.

\section{RÉSUMÉS}

A partir de la lecture de trois définitions d'instruments de musique, cet article aborde les équivoques propres aux dictionnaires de langue. Sous couvert de langue reçue, ces instruments normatifs, peu formalisés, pratiquent l'oubli des dires passés et fonctionnent essentiellement par effets d'intertextualité : rôle des sources d'emplois, des domaines, des traditions langagières... Plus généralement nous proposons ici d'étudier le dictionnaire comme un lieu de mise en contact d'univers discursifs disjoints.

This article deals with equivocations in dictionaries through the analysis of three definitions of musical instruments. These hardly formalized, normative tools, while recording the descriptive use of language, ignore its original contextual usage. Consequently, they essentially operate as instruments for interlanguage through the role of fields and traditions of usage. More generally speeking the article will examine the role of the dictionary as a place of interaction between disconnected discourses.

\section{AUTEUR}

\section{FRANCINE MAZIÈRE}

